\title{
Augmenting Accessibility Guidelines with User Ability Rationales
}

\author{
Christophe Ponsard ${ }^{1}$, Pascal Beaujeant ${ }^{2}$, and Jean Vanderdonckt ${ }^{2}$ \\ ${ }^{1}$ CETIC Research Centre, Gosselies, Belgium \\ ${ }^{2}$ Louvain School of Management, Université Catholique de Louvain, \\ Louvain-la-Neuve, Belgium \\ christophe.ponsardacetic.be, \\ \{pascal.beaujeant, jean.vanderdonckt\} @uclouvain.be
}

\begin{abstract}
Designing accessible web sites and more generally Internetconnected devices remains a challenging task nowadays. A number of guidelines (such as the WCAG2) are now widely available and recognised. To better cope with the quickly evolving technological landscape, these guidelines are also being formulated in technology-neutral terms. However this is still leaving the user dimension largely implicit, which makes it difficult to understand exactly which kind of user a given website is hindering.

This paper describes how to capture and use rational links between guidelines and user capabilities/impairments by combining a set of complementary models (user, task, user interface, guidelines). The process of building those accessibility rationales relies upon available user and guidelines ontologies and also on obstacle identification and resolution techniques borrowed from the requirements engineering domain. This resulting enriched guidance enables a number of interesting new scenarios to better help web developers, analyse guidelines or make comparisons between guidelines.
\end{abstract}

Keywords: Accessibility, Assessment, Web, User-Model, Task Model, Ontology, Guidelines, WCAG.

\section{Introduction}

Keeping the web accessible to all and especially to people with disabilities has become fundamental given the importance the web as gained in many aspects of life such as education, employment, commerce, health care, government, leisure, and more. Considering sight and hearing impairments which are among the most problematic when considering web usage, about 6.4 percent of the population age 15 years and older and more than $20 \%$ of the population over 65 is concerned in the US, according to a 2010 report [2]. Those figures can be transposed to other developed countries and are far higher in developing countries.

Applying accessibility principles is also widely recognised as enhancing the overall usability experience for everyone whether or not suffering impairment. 
Accessibility standards such as the WCAG [13] or section 508 [11] are widely recognised and formulated as practical guidelines to ease adoption.

Despite those facts, recent reports keep showing that the level of accessibility remains very low. For example, a 2008 European Commission study highlighted that only $2.6 \%$ of key public and commercial websites in Member States were accessible, while only $5.3 \%$ of government websites were accessible [6]. Explaining the gap between the need and the current lack of accessible websites is not easy and multiple factors are involved. The fast pace of technical evolution does not help but beyond that, one of the key reasons frequently mentioned is the lack of information and training of web designers.

Accessibility guidelines have quite matured and now clearly separate the accessibility principles and guidelines from specific techniques to enforce them in specific technologies [13]. However, while web designers are well armed to face "how" to apply the principles, the support to help them understand "why" they need to apply specific guidelines is still largely missing; e.g. the WCAG2, focussed upon in this paper, does not explicitly relate the need of captions in video content with deafness.

In order to provide better support for the web designer, this paper proposes to enhance the existing guidelines with such rationales. We show how to systematically produce them based on the existing guidelines combined with user impairment ontologies and user interface modelling techniques abstracting from the pure implementation level (such as abstract user interfaces and tasks models). In order to represent and reason about the accessibility related knowledge, we rely on semantic web techniques like RDF/OWL representations, queries and ontology interconnections [10]. Those techniques also enable an access as open linked data.

The structure of this paper follows our research approach. First, in Section 2, we survey the available models and related ontologies which can be exploited and connected to better support the design process. Section 3 then details how additional links can be inferred to bridge important gaps such as the rationales between guidelines and user impairments. Section 4 then illustrates a number of scenarios on how the enriched guidelines bring added value. Section 5 reviews some related works; and finally section 6 concludes by highlighting how this work can be further developed.

\section{Survey of Accessibility Related Ontologies and Models}

This section presents the characteristics (strength/limitations) of existing accessibility and impairment ontologies as well as relevant User and UI modelling techniques.

\subsection{Accessibility and Impairement Ontologies}

Web Content Accessibility Guidelines. WCAG guidelines are expressed as principles in four major categories: perception, operability, understandability and robustness. Those are refined into 12 major guidelines which are then supported by specific techniques, either generic or specific to a technology. Related checks are also defined to keep the traceability between these guidelines. Its structure can be represented as a goal refinement tree depicted in Figure 1 and explained in more details in section 2.2. 


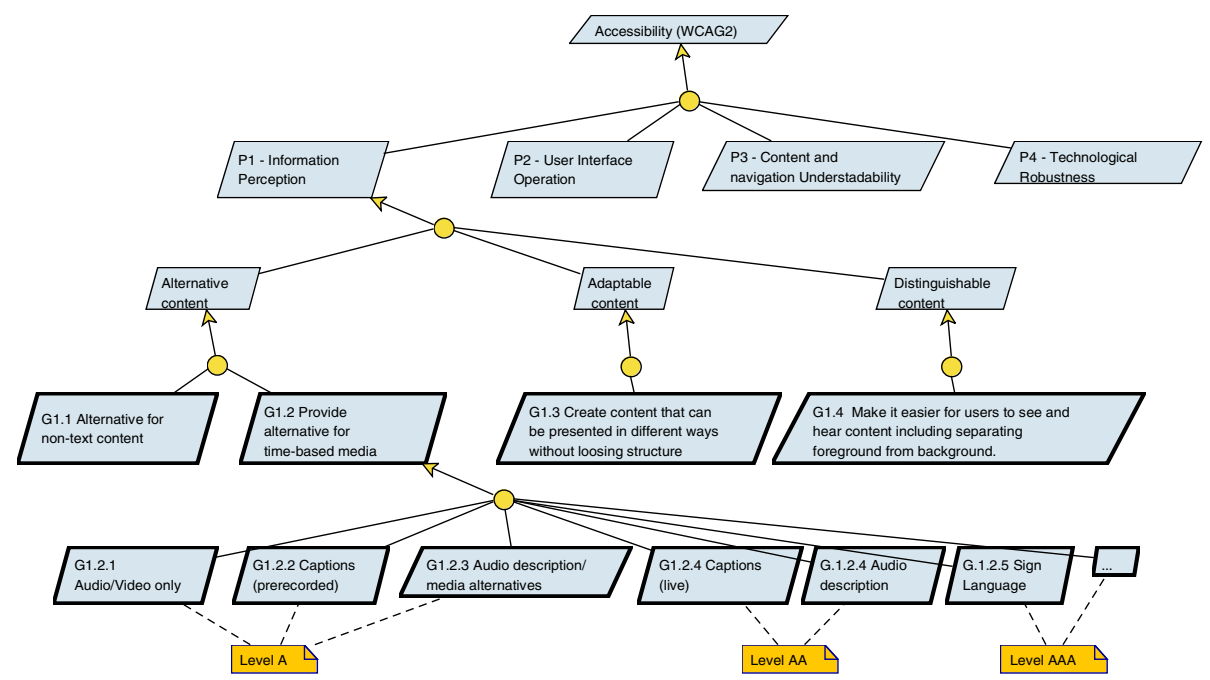

Fig. 1. Partial goal model for WCAG2 guidelines

The guidelines are mostly available as well-organised structured hypertext on the W3C website. The AEGIS project has also made them available as ontology under the OWL format which can be used for semantic processing [1]. Figure 2 illustrates this ontology in the Open Source Protégé tool.

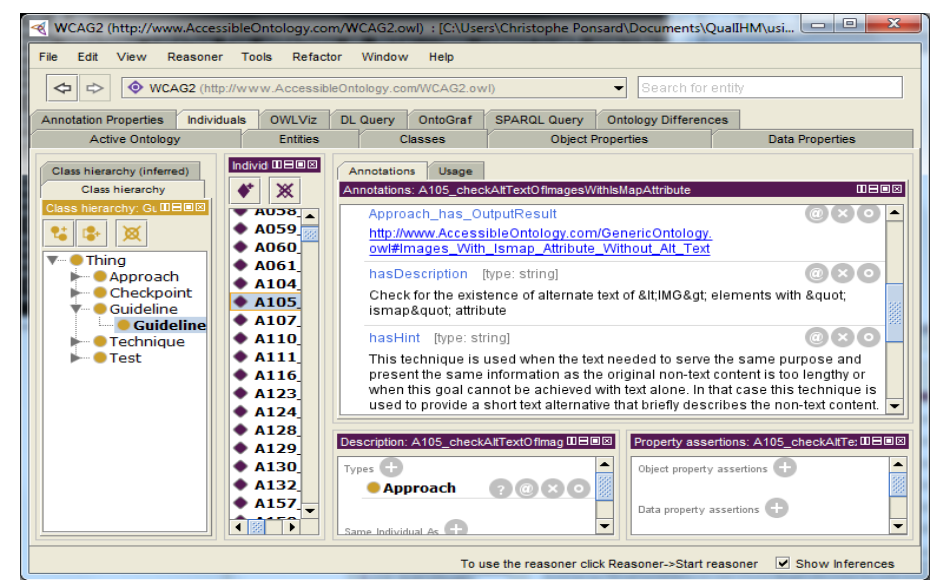

Fig. 2. WCAG2 Ontology

International Classification of Functioning, Disability and Health (ICF) [5]. It is a universal classification of disability and health for the definition, measurement and policy formulations in health and health-related sectors. The naming reflects its philosophy oriented to the measuring functioning in society, no matter what the reason for one's impairments. This is reflected by its conceptual structure represented in 


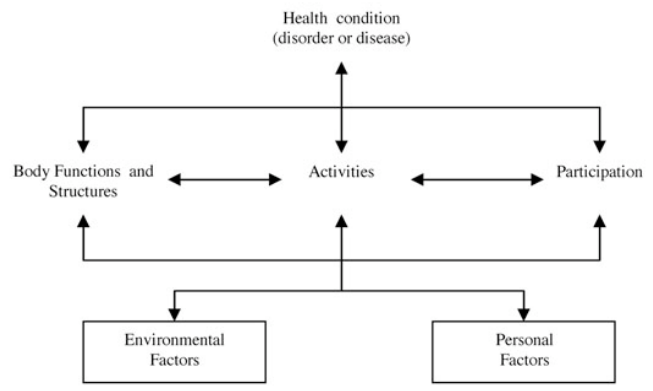

Fig. 3. ICF Concepts and Relationships

Figure 3. It is therefore relevant to consider this classification also in the specific context of IT usage and web accessibility. An extensive OWL ontology has been released by the BioPortal, composed of about 1600 classes and 3250 individuals [3].

\subsection{Models}

User Interface Models provide representations of the user interaction with a computer program or another reactive device with the purpose of supporting the UI design and analysis process. Different aspects of human-machine interaction can be captured by a set of the following complementary models and are supported by specification languages like UsiXML [12].

- Task model enables the description of high-level user requirements in terms of activities to be performed by the user and/or by the system in order to reach some goal [14]. They have a hierarchical graphical syntax with a well-defined semantics.

- Abstract UI model defines interaction spaces grouping subtasks according to various criteria and independently of any context/modality of use.

- Concrete UI model define widgets layout and navigation. While making the Look \& Feel explicit, it is still a mock-up rendered in a non-operational environment.

Goal models are used in the larger context of requirements engineering of system [15] while task models are more specifically targeting UI design. Goals capture properties to be achieved by users together with the systems. Goals can be refined hierarchically and can be seen to some point as a generalisation of tasks. Goals support reasoning about obstacles and conflict by taking into account known domain properties (in our context: UI characteristics and user impairments).

\section{$3 \quad$ Producing Guidelines Rationales}

In this section, we present the process to generate accessibility guideline rationales, based on existing techniques used among other in requirements engineering such as obstacle analysis and abduction techniques based on domain knowledge [15]. This rationale generation process is depicted in Figure 4 and relies on the following steps: 
1. Content types are extracted from the models, possibly at different level of abstraction (e.g. task model, abstract UI model if available or concrete UI)

2. Obstacle analysis is performed based on the user capability ontology (e.g. ICF).

3. Matching is made with existing guidelines provided by the guideline library (e.g. WCAG2).

The process can be complemented by a bottom-up phase, starting from the existing guidelines and attaching them to obstacle or completing existing obstacle trees.

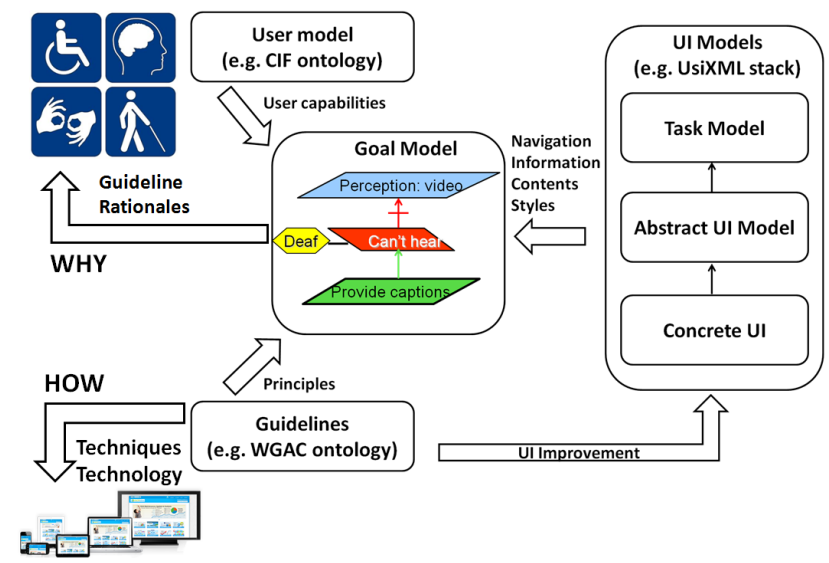

Fig. 4. Rationale Generation Approach

This process can be applied systematically by using the rich ICF and WCAG ontologies identified in the section 2. The captured knowledge can then be bundled into a merged ontology enriched with the extra links illustrated in the following example:

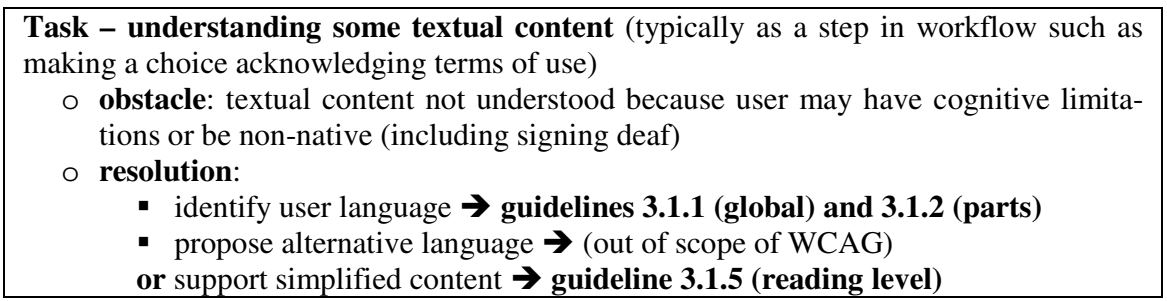

Fig. 5. Example of rationale generation

The systematic application of the above process revealed two interesting points. First, it acts as a completeness check to detect partly addressed impairments in some usage context. Second, some models seem to relate more specifically to certain functional impairment categories (see Table 1). This can help having a special focus when building those models or even support the decision to build such models. 
Table 1. Impacted functions in relation with UI models

\begin{tabular}{lll}
\hline UI model & UI concepts (added from previous level) & Impacted functions \\
\hline Task & Information flow, navigation flow & Cognitive \\
Abstract UI & Controls, content types & Motor, Vision, Hearing \\
Concrete UI & Look and Feel (styles, colors) & Vision \\
\hline
\end{tabular}

\section{Usage Scenarios}

The availability of the guideline rationales enables new usage scenarios for different target groups (web designers, developers, assessors,...). The scenarios reported here were identified together with Anysurfer (http://www.anysurfer.be), a major association active in accessibility assessment and training. Some subjective validation elements are also reported.

\subsection{Providing User-related Explanations for Web Designers}

Currently, the application of guidelines produces a global ranking such as A, AA, AAA with limited explanations in terms of passed/failed checks. Problems can be traced to guidelines and corrective actions. The rational information enables to:

- state which kind of user is impacted by a failed check. The feedback is this helps web designers to better identify the type of impaired users and to better understand and to apply the relevant parts of guidelines.

- provide finer grained assessment going by functional impairment going beyond "black box" A/AA/AAA labels. Some development can for example target a specific user group for which extra requirements have to be implemented.

This support to web designers can be part of an accessibility assessment report but larger gain is expected when it is applied at design stage.

\subsection{Aligning Guidelines for Web Designers}

This paper is focusing on the WCAG2 which are the major reference guidelines. There are however other guidelines such as section 508 in the US and BS 8878:2010 Web accessibility in UK. For global websites or websites in countries transitioning to WCAG, it is more effective to be able to compare between guidelines rather than performing multiple independent assessments.

Table 2. Guideline Alignment example

\begin{tabular}{llll}
\hline ICF & Obstacle to address & WCAG2 & Section 508 \\
\hline b.21021 Seing fion: colour vision & Colour only information & G1.4.1 & $1194.21 \mathrm{i}$ \\
b.21022 Seing fion: contrast sensi- & Contrast enhancement & G1.4.3 & $1194.21 \mathrm{~g}$ \\
tivity & & G1.4.6 & \\
\hline
\end{tabular}


The proposed process can be applied to multiple guidelines with the same structure, resulting in a natural alignment of guidelines as illustrated in Table 2.

\subsection{Checking Gaps in the Guidelines for Accessibility Working Groups}

As mentioned in Section 3, the completeness check can point out some obstacles not addressed by the considered guidelines. The validation with accessibility assessors revealed some possible reasons for this: the topic might be related to usability rather than accessibility, or corrective measures might have been considered too advanced/costly to implement. In all cases, it was found interesting to report this information for helping in the future evolution of the guidelines. Some issues can also be reported to web developers as recommendations going beyond the current guidelines.

\section{$5 \quad$ Related Work}

Applying ontologies to accessibility has already been proposed. For instance, [16] shows how they help in addressing limitations of natural language but without referring to specific guidelines. This is now a recognised approach as shown in Section 2.

The idea to connect ontologies has been investigated in [7]. A generic accessibility pattern connecting user, capability, interface element and information has been proposed and instantiated to various examples (memory/recall, perception). The work however does not explore the use of renown and standard ontologies like the ICF and WCAG as proposed in our work.

An alternative approach proposed in [9] is to build a specific ontology formally describing the whole information about user's impairments, and the available interface characteristics. This allows a personalised accessibility assessment but the presented scenario does not seem to support the detection of partly covered impairments. We also believe it is better to avoid mixing ontologies addressing different domains to ease future evolution, for example considering different sets of guidelines.

The project ACCESSIBLE developed a harmonised methodology for measuring accessibility, including guideline alignment [8]. Though quite similar, that work looks ICF-driven (top-down) while our approach can also be guidelines driven (bottom-up). The work also covers the alignment of guidelines such as WCAG1/2 and section 508 .

AEGIS project developed an Open Accessibility Framework relying on ontologies [1]. It supports the mapping from requirements/constraints of users to characteristics not only of web applications but also desktop and mobile applications. The AEGIS generic accessible user interaction model shares similarities with our work although it is more directed towards personal customization scenarios.

\section{Conclusion and Perspectives}

The paper shows how accessibility guidelines can usefully be extended to better support accessibility rationales providing better explanation why specific guidelines 
are required by linking them to impairments. A number of interesting scenarios exploiting these rationales have been identified and investigated.

At this point, the resulting combined ontology is still partial but available from http://www.accessible-it.org/ontologies. On-going work is to enrich them and achieve integration with existing frameworks in order to conduct validation experiment inside a local cluster of web SMEs. We also plan to investigate more specific user capability models such as the DSM-5 [3] dealing with cognitive disorders.

Acknowledgement. This work was funded by the QuallHM project (grant $\mathrm{nr}$ 1217570) as part of the collective research programme of the Walloon region. We warmly thank the Belgian AnySurfer association and the reviewers for their insightful feedback.

\section{References}

1. AEGIS Project. Open Accessibility Everywhere, http : / /www . aegis-project. eu

2. Americans With Disabilities: 2010, report from the US Census Bureau (July 2012)

3. American Psychiatric Association, DSM-5 Development, http://www. dsm5 . org

4. BioPortal, ICF Ontology, http://bioportal.bioontology.org/ontologies/1411

5. International Classification of Functioning, Disability and Health (ICF), http: / / www . who. int/icidh

6. European Commission, Web-accessibility in European countries: level of compliance with latest international accessibility specifications, notably WCAG 2.0, and approaches or plans to implement those specifications, SMART 2008/0068 (2008)

7. Karim, S., Latif, K., Tjoa, A.M.: Providing universal accessibility using connecting ontologies: A holistic approach. In: Stephanidis, C. (ed.) HCI 2007. LNCS, vol. 4556, pp. 637-646. Springer, Heidelberg (2007)

8. Mourouzis, A., Kastori, G.-E., Votis, K., Bekiaris, E., Tzovaras, D.: A Harmonised Methodology towards Measuring Accessibility. In: Stephanidis, C. (ed.) Universal Access in HCI, Part I, HCII 2009. LNCS, vol. 5614, pp. 578-587. Springer, Heidelberg (2009)

9. Oikonomou, T., Kaklanis, N., Votis, K., Tzovaras, D.: An Accessibility Assessment Framework for Improving Designers Experience in Web Applications. In: Stephanidis, C. (ed.) Universal Access in HCI, Part I, HCII 2011. LNCS, vol. 6765, pp. 258-266. Springer, Heidelberg (2011)

10. OWL Web Ontology Language Reference, http: / /www.w3 . org/TR/owl-ref

11. US Government, Section 508, https : / / www . section508.gov

12. Vanderdonckt, J., Limbourg, Q., Michotte, B., et al.: USIXML: A User Interface Description Language for Specifying Multimodal User Interfaces. In: W3C Workshop on Multimodal Interaction, Sophia Antipolis (2004)

13. Web Content Accessibility Guidelines (WCAG), http: / / www . w3 . org/TR/WCAG/

14. W3C, MBUI - Task Models, Working Draft (2012), http: / /www.w3 .org/TR/task-models

15. van Lamsweerde, A.: Requirements Engineering: From System Goals to UML Models to Software Specifications. Wiley (2009)

16. Xiong, J., Farenc, C., Winckler, M.: Towards an Ontology-Based Approach for Dealing with Web Guidelines. In: Hartmann, S., Zhou, X., Kirchberg, M. (eds.) WISE 2008. LNCS, vol. 5176, pp. 132-141. Springer, Heidelberg (2008) 